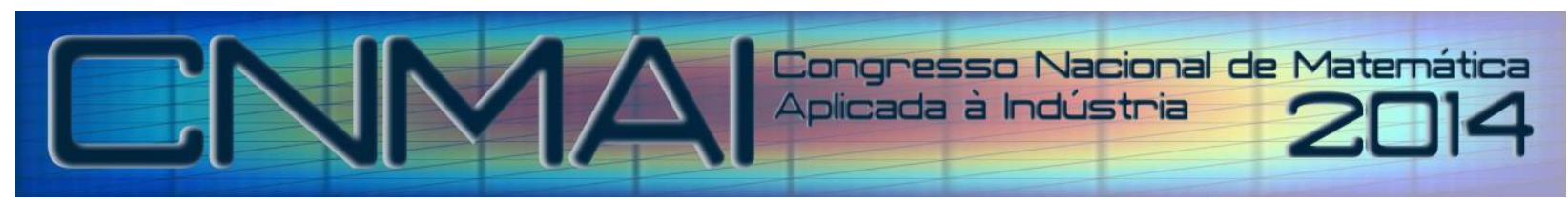

18 a 21 de novembro de 2014, Caldas Novas - Goiás

\title{
ANÁLISE DE DIFERENTES ESTIMADORES DE ERRO A POSTERIORI APLICADOS A PROBLEMAS BIDIMENSIONAIS UTILIZANDO REFINO H-ADAPTATIVO
}

\author{
Jéderson da Silva, dasilva.jederson@gmail.com ${ }^{1}$ \\ Francielly Elizabeth de Castro Silva, franciellye.castro@gmail.com ${ }^{1}$ \\ Jucélio Tomás Pereira, jucelio.tomas@ufpr.br ${ }^{1}$ \\ João do Carmo Lopes Gonçalves, joao.carmo @ ifpr.edu.br ${ }^{1,2}$
}

\begin{abstract}
${ }^{1}$ Universidade Federal do Paraná - Programa de Pós-Graduação em Engenharia Mecânica (PG-Mec) - Bloco IV do
Setor de Tecnologia, Centro Politécnico da UFPR, Bairro Jardim das Américas, Curitiba, Brasil.

${ }^{2}$ Instituto Federal do Paraná - Campus Paranaguá, Bairro Porto Seguro, Paranaguá, Brasil.
\end{abstract}

\begin{abstract}
Resumo: O desenvolvimento de eficientes processos de geração de malhas adaptativas para análises de elementos finitos tem sido foco de intensivas pesquisas nas últimas décadas. Uma confiável estimativa de erro de aproximação deste método numérico é necessária para guiar de maneira adequada um processo adaptativo. O presente trabalho discute a análise de erros de aproximação, suas estimativas e a adaptatividade da malha quando da aplicação do Método dos Elementos Finitos (MEF) em problemas bidimensionais utilizando o elemento triangular linear CST (Constant Strain Triangle). A estimativa dos erros a posteriori baseia-se na recuperação das derivadas de ordem superior e na norma de energia. São utilizados os seguintes estimadores: estimador fundamentado na Média Nodal Simples (MNS), estimador Zienkiewicz e Zhu (ZZ) e estimador Superconvergent Patch Recovery (SPR). Em todos os problemas abordados emprega-se como medida da qualidade dos estimadores o conceito de indices de efetividades. Com o cálculo do erro em cada elemento, é utilizado um processo h-adaptativo para gerar malhas de elementos finitos adequadas ao problema em estudo, as quais objetivam um erro aproximadamente uniforme e não superior ao erro admissível. Discute-se a teoria inerente à estimativa de erro e os princípios básicos do processo h-adaptativo adotado. Através de exemplos numéricos é realizada uma análise comparativa entre os diferentes estimadores e suas eficiências no contexto de um processo h-adaptativo.
\end{abstract}

Palavras-chave: Método dos Elementos Finitos, H-adaptatividade, Estimadores de erro a posteriori, Estimador Superconvergent Patch Recovery, Estimador de Zienkiewicz e Zhu (ZZ).

\section{INTRODUÇÃ̃O}

A aplicação de métodos numéricos como MEF, Método dos Elementos de Contorno, Método das Diferenças Finitas, para solução e análise de problemas complexos de engenharia tem sido cada vez mais difundida e incentivada por apresentar várias vantagens como redução do tempo de cálculo, precisão e automação do processo numérico. Paralelamente ao desenvolvimento desses métodos surgem intensas pesquisas em relação à estimativa dos erros ocasionados pela aproximação da solução e a geração automática de malhas adequadas aos problemas em estudo.

Em geral, ancorado em estimadores de erro confiáveis e estratégias adaptativas automatizadas busca-se gerar malhas de elementos finitos ajustadas a cada problema, diminuindo cada vez mais os requisitos quanto a um grande conjunto de conhecimentos sobre o problema estudado e da experiência do projetista.

Uma das principais categorias de estimativas de erro em elementos finitos é a estimativa de erro a posteriori (Ainsworth e Oden, 2000), a qual é utilizada nesse trabalho. Estimadores de erro a posteriori utilizam a própria solução numérica para realizar a estimativa dos erros e podem ser empregados para desenvolver algoritmos de malhas adaptativas uma vez que as mesmas permitem computar o tamanho dos erros locais (Prudhomme et al., 2003).

Procedimentos adaptativos procuram ajustar automaticamente a malha de uma maneira que se obtenha uma solução com uma precisão especificada. Em geral o procedimento é iniciado considerando uma malha grosseira e, com as estimativas dos erros locais, são realizados ajustes de forma a obter uma malha adequada ao problema. O corrente trabalho utiliza uma estratégia h-adaptativa para geração da malha, na qual os elementos procedentes da discretização tem seus tamanhos alterados, permanecendo-se inalterável o grau do polinômio empregado (Zhu e Zienkiewicz, 1988). 
Nesse contexto, o corrente trabalho aborda a análise de erros de aproximação, suas estimativas e a adaptatividade da malha quando da aplicação do MEF em problemas planos de condução térmica, empregando para guiar o processo adaptativo três diferentes estimadores de erro a posteriori: Método das Médias Nodais Simples (MNS), Estimador de Zienkiewicz e Zhu (ZZ) e Estimador Superconvergent Patch Recovery (SPR).

\section{ESTIMATIVA DE ERRO NO MÉTODO DOS ELEMENTOS FINITOS}

De forma a descrever de maneira adequada o problema e o método numérico adotado (MEF), é conveniente uma breve discussão sobre a formulação do problema de equilíbrio e a correspondente discretização. Nesse caso, considerando um problema linear de condução de calor definido em um domínio $\Omega$ e submetido unicamente a condições de contorno de Dirichlet homogêneas, tem-se que sua forma forte pode ser posta como (Reddy, 2006)

$$
\begin{aligned}
& -\nabla(k \nabla T)=f \quad \text { em } \Omega \\
& T=0 \quad \text { em } \Gamma_{T},
\end{aligned}
$$

onde $T$ é o campo de temperaturas, $k$ a matriz de condutividade térmica, $f$ a fonte interna de calor, $\Gamma_{T}$ o contorno e $\nabla$ é o operador gradiente, o qual pode ser expresso por

$$
\nabla(.)=\frac{\partial(.)}{\partial x} \mathrm{i}+\frac{\partial(.)}{\partial y} \mathbf{j}
$$

Durante o processo de discretização por elementos finitos, aproxima-se a solução analítica $T$ por

$$
T \approx \hat{T}=\sum_{e l=1}^{N_{e l}} \sum_{n o=1}^{N_{n o}} t_{n o}^{e l} \Psi_{n o}^{e l} \equiv \sum_{i=1}^{Z} t_{i} \Psi_{i}
$$

onde $\hat{T}$ é a solução de aproximação por elementos finitos sobre o domínio, $N_{e l}$ é o número total de elementos na malha, $Z$ é o número total de nós globais, $N_{n o}$ é o número de nós em um elemento, $t_{n o}^{e l}$ indica o valor da temperatura no elemento $e l$ do nó no do elemento, $t_{i}$ denota o valor de $\hat{T}$ no nó global i e $\Psi_{i}$ denota a função de interpolação global associada ao nó global i. Aplicando-se o Método dos Resíduos Ponderados de Galerkin, obtêm-se um sistema matricial de equações que relaciona o vetor de temperaturas nodais $t$ e o vetor de fluxos externos nodal consistente $(F)$ com a função de fonte interna de calor $f$, através da matriz de rigidez $K$, na forma

$$
K t=F .
$$

Considerando cada elemento, a relação elementar entre as temperaturas nodais e os correspondentes gradientes de temperatura $\hat{G}_{T}$ e fluxos de calor $\hat{q}$ é dada por

$$
\hat{G}_{T}=B t_{n o}^{e l} \quad e \quad \hat{q}=-k \hat{G}_{T},
$$

onde a matriz $B$ contém as derivadas das funções de interpolação linear em relação às coordenadas globais x e y, enquanto que a matriz $k$ contém as características de condutividade térmica do material.

Como a solução obtida $\hat{T}$ não é a solução exata do problema exposto pela Eq. (2.1), é de extrema importância responder a seguinte questão: como o erro pode ser medido, controlado e efetivamente minimizado?

Segundo Zienkiewicz e Taylor (2000) o erro de aproximação $(e)$ depende da malha adotada e pode ser definido como a diferença entre a solução analítica e a solução aproximada obtida pelo MEF, sendo, portanto, expresso por

$$
e=T-\hat{T}
$$

Com o objetivo de obter um valor escalar que meça a função erro em um sentido global para a corrente malha, adota-se uma medida em forma de norma. Uma das normas mais utilizadas é a norma em energia que, para o problema em análise, pode ser posta como

$$
\|e\|^{2}=\int_{\Omega}(q-\hat{q})^{T} k^{-1}(q-\hat{q}) d \Omega
$$


Uma maneira conveniente de quantificar o erro medido na norma em energia é o erro relativo percentual em energia, $\eta$, descrito pela razão entre o erro na norma em energia e a energia total acumulada no domínio, $\|u\|$, dado por

$$
\eta=\frac{\|e\|}{\|u\|} 100
$$

onde $\|u\|$ é definido como

$$
\|u\|^{2}=\int_{\Omega}(\nabla T)^{T} k(\nabla T) d \Omega .
$$

Porém, como a solução analítica $T$ não é conhecida, busca-se empregar uma solução com uma taxa de convergência superior a solução gerada diretamente por MEF $\hat{q}$, a qual é denominada de solução recuperada $q^{*}$. Assim, o estimador de erro segundo a norma em energia pode ser redefinido como

$$
\left\|e^{*}\right\|^{2}=\int_{\Omega}\left(q^{*}-\hat{q}\right)^{T} k^{-1}\left(q^{*}-\hat{q}\right) d \Omega
$$

A qualidade dos estimadores de erro é medida pelo índice de efetividade $\theta$, descrito pela razão entre o valor do erro estimado pela equação (2.10) e o valor verdadeiro do erro, o qual é obtido pela Eq. (2.7). Em casos onde não se conhece a solução analítica do problema, esta é substituída por uma solução obtida de uma malha bem refinada. Nesse contexto, o que espera é um estimador de erro assintoticamente exato, ou seja, que seu índice de efetividade tenda a unidade quando o erro estimado tenda a zero. A qualidade do processo de recuperação adotado determina a qualidade do estimador se, sendo ele assintoticamente exato, somente se a solução recuperada convergir para a solução analítica a uma taxa superior a solução de MEF (Zienkiewicz e Taylor, 2000).

\subsection{Método das Médias Nodais Simples}

O Método das Médias Nodais Simples é um método clássico e de simples implementação. Este consiste em, após o cálculo do fluxo de calor $\hat{q}$ em cada elemento conectado a cada nó, e sabendo que para um nó compartilhado por $\mathrm{Nel}_{n o}$ elementos existem, via de regra, $\mathrm{Nel}_{n o}$ diferentes valores de $\hat{q}$, pode-se estimar a média nodal como (Cook et al., 2002)

$$
(q)_{m e d}=\frac{1}{N e l_{n o}} \sum_{i=1}^{N e l_{n o}}(\hat{q})_{i} .
$$

Um campo suavizado e contínuo entre os elementos pode ser construído através dos fluxos de calor médios nodais utilizando as mesmas funções de interpolação $\Psi$ utilizadas para interpolar as temperaturas nodais. Dessa forma, a parcela de um campo de fluxos de calor suavizados sobre um elemento pode ser escrita como

$$
q_{\alpha}^{*}=\Psi q_{n_{\alpha}}^{*},
$$

onde $q_{\alpha}^{*}$ é qualquer componente do fluxo de calor suavizado $\left(q_{x}\right.$ ou $\left.q_{y}\right)$ e $q_{n_{\alpha}}^{*}$ é o vetor de médias nodais de fluxos de calor para o elemento em questão.

\subsection{Estimador de erro de Zienkiewicz e Zhu (ZZ)}

Basicamente, o clássico Estimador ZZ consiste em uma abordagem similar à adotada pelo método das médias nodais, ou seja, estima o erro através de um campo de fluxos de calor suavizados contínuo ao longo de todo o domínio, o qual é obtido a partir da interpolação dos valores nodais utilizando as mesmas funções de interpolação empregadas para interpolar as temperaturas nodais (Eq. 2.12). A diferença básica entre os estimadores é como recuperar os valores nodais dos fluxos de calor $q_{n}^{*}$. Nesse caso, Zienkiewicz e Taylor (1989) calculam os valores nodais dos fluxos como

$$
q_{n}^{*}=M^{-1}\left(\int_{\Omega} \Psi^{T} \hat{q} d \Omega\right)
$$


onde $M$ é conhecida como a matriz de massa, expressa por

$$
M=\left(\int_{\Omega} \Psi^{T} \Psi d \Omega\right) .
$$

\subsection{Método Superconvergent Patch Recovery (SPR)}

No MEF existem pontos no interior do domínio de cada elemento que possuem uma característica denominada superconvergência. Ou seja, os valores dos gradientes ou fluxos de calor apresentam um erro que decresce mais rapidamente do que em outras regiões do elemento (Zienkiewicz e Taylor, 2000). A localização desses pontos para fluxos de calor coincidem com os pontos de Gauss usados para integrar exatamente a matriz de rigidez. A ideia básica do Método SPR é similar à empregada pelo MNS e pelo ZZ. Ou seja, parte da Eq. (2.12) para gerar um campo de fluxos de calor suavizados, diferindo somente pela maneira como os valores nodais $q_{n}^{*}$ são obtidos.

De modo geral, o SPR obtém um polinômio que aproxima os fluxos de calor e define os valores recuperados nos nós utilizando um ajuste por mínimos quadrados de soluções conseguidas nos pontos superconvergentes. Para tal utiliza o conceito de padrões ( $p a t c h$ ) onde, ao invés de determinar o polinômio aproximado ao longo de todo o domínio, obtém o polinômio que aproxima os fluxos de calor dentro de padrões. Esses padrões são definidos pelo anexo de elementos que estão conectados a um mesmo nó.

Assim, de acordo com Zienkiewicz e Taylor (2000), para cada componente $\hat{q}_{i}$ de $\hat{q}$ escreve-se a solução recuperada como

$$
q_{n_{i}}^{*}=p(x, y) a=[1, x, y] a,
$$

sendo os coeficientes do polinômio dados por

$$
a=\left[a_{1}, a_{2}, a_{3}\right]^{T} .
$$

Estes coeficientes são obtidos pela minimização da distância quadrática $(\Pi)$ entre este polinômio e os valores de fluxo de calor nos pontos de Gauss dos elementos que compõe o padrão para o nó.

Uma vez determinados os coeficientes do polinômio, substituem-se as coordenadas do nó central do padrão na Eq. (2.15) para obter os valores nodais de fluxos de calor recuperados no elemento $q_{n}^{*}$. Com os valores nodais emprega-se a Eq. (2.12) para obter a distribuição dos fluxos de calor ao longo de todo o conjunto de elementos.

Cabe ressaltar que para que a técnica funcione de maneira efetiva é necessário que o número de pontos superconvergentes presentes no padrão seja igual ou maior que o número de coeficientes do polinômio $a$ (Cook et al., 2002). Em caso contrário, Zienkiewicz e Zhu (1992) recomendam que se empregue o polinômio aproximador do padrão mais próximo.

\section{MALHA H-ADAPTATIVA}

Utilizando qualquer um dos estimadores de erro descritos acima, é possível quantificar o erro localmente para cada elemento. Dessa maneira, seguindo um processo h-adaptativo, ou seja, um processo no qual o número e o tamanho dos elementos se ajustam para satisfazer um determinado erro admissível, é possível obter, a partir de uma malha grosseira e não adequada, uma malha melhorada, orientada pelas estimativas dos erros locais.

Neste caso, espera-se que o erro relativo percentual em energia $\eta$ para uma malha $m$ seja menor ou igual a um valor admissível $\bar{\eta}$. Ou seja, dada uma malha $m$, onde

$$
\eta_{m}>\bar{\eta}
$$

busca-se uma malha $m+1$ tal que

$$
\eta_{m+1} \leq \bar{\eta} .
$$

Como critério de malha ótima, este trabalho emprega a condição de equidistribuição do erro em energia (Zienkiewicz e Zhu, 1987, Wiberg e Abdulwahab, 1997, Zienkiewicz e Taylor, 2000 e Castellazzi et al., 2010). Para uma estimativa a posteriori do erro, um limite superior para o mesmo pode ser posto como

$$
\|e\| \leq C h^{\min (p, \lambda)},
$$


onde $\|$.$\| representa a norma em energia, C$ é uma constante, $h$ representa o tamanho de um elemento finito, $p$ é a ordem polinomial do elemento e $\lambda$ é um parâmetro que define o tipo de singularidade. Considerando que esta equação de projeção para o erro é válida para a malha corrente $m$ e para a próxima malha $m+1$, obtém-se uma estimativa para o novo tamanho do elemento $h_{\text {new }}$ na forma

$$
h_{\text {new }}=\frac{h_{\text {old }}}{\xi^{1 / p}} \quad \text { onde } \quad \xi=\frac{\|e\|_{\text {el,old }}}{\|e\|_{\text {el,new }}},
$$

sendo $\|e\|_{e l, o l d}$ o erro para um elemento el na malha corrente com o tamanho de elemento $h_{\text {old }}$, $\|e\|_{\text {el, new }}$ o erro para um elemento $e l$ na malha $m+1$. Neste caso, $\xi$ é um parâmetro de refino que indica se a malha será (para $\xi>1$ ) ou não (para $\xi<1$ ) refinada. Considerando a condição de equidistribuição, o erro máximo admissível para cada elemento pode ser calculado como

$$
\|e\|_{\text {el,new }}=\bar{\eta}\left(\frac{\|u\|^{2}}{N e l}\right)^{1 / 2} \approx \bar{\eta}\left(\frac{\|\hat{u}\|^{2}+\left\|e^{*}\right\|^{2}}{N e l}\right)^{1 / 2},
$$

onde $\left\|e^{*}\right\|$ é o erro total na norma em energia calculado por

$$
\left\|e^{*}\right\|^{2}=\sum_{e l=1}^{N e l}\left\|e^{*}\right\|_{e l}^{2}=\sum_{e l=1}^{N e l}\left\|u^{*}-\hat{u}\right\|_{e l}^{2}
$$

e $\|u\|$ é a medida da energia total acumulada. Supondo que a solução analítica não é conhecida, utiliza-se para o cálculo de $\|u\|$ o conceito de que o erro é ortogonal a solução aproximada (Ciarlet, 2002). Nesse caso,

$$
\|u\|^{2}=\|\hat{u}\|^{2}+\left\|e^{*}\right\|^{2}
$$

sendo $\|\hat{u}\|^{2}$ calculado por

$$
\|\hat{u}\|^{2}=\int_{\Omega}\left(\hat{G}_{T}\right)^{T} k\left(\hat{G}_{T}\right) d \Omega .
$$

Com o objetivo de controlar o crescimento desordenado dos elementos, o qual pode gerar uma baixa qualidade da malha obtida, é introduzido um fator de amortecimento sempre que $\xi<1$, ou seja, quando ocorre desrefino. Neste caso, aplica-se (Fancello e Feijóo, 1992)

$$
\xi \rightarrow \xi+\frac{(1-\xi)}{2}
$$

\section{EXEMPLOS NUMÉRICOS}

De modo a comparar a eficiência dos diferentes estimadores de erro aplicados a análises adaptativas, nesta seção são apresentados alguns exemplos numéricos aplicados ao problema plano de condução térmica. Todos os resultados utilizam o elemento CST e empregam os processos de estimativa de erro e de adaptação da malha descritos anteriormente. A estrutura computacional é programada utilizando o software Matlab e a geração completa da malha refinada com os parâmetros calculados pelo processo adaptativo é realizada empregando o software ARANHA (Fancello et al., 1990).

O problema de condução térmica solucionado difere em cada caso pela fonte interna de calor aplicada, sendo algumas características comuns a todos os exemplos. Nesse caso, pode-se enunciar o problema de condução térmica através da Eq. (2.1), onde o domínio global é definido como $\Omega=[0,1]^{2}$.

A medida da eficiência dos diversos estimadores aplicados ao processo adaptativo é realizada através da comparação entre variáveis que caracterizam o processo e a malha em análise como: o número de elementos, o número 
de graus de liberdade, o erro relativo percentual em energia e o índice de efetividade. Essa comparação é efetuada, em cada exemplo apresentado, confrontando os resultados dos próprios estimadores em análise. Em alguns casos é possível a comparação dos resultados obtidos com outras abordagens apresentadas na literatura.

\subsection{Problema 1}

Neste exemplo, a fonte interna de calor aplicada é selecionada tal que a solução analítica é dada por (Castellazzi et al., 2010 e Zienkiewicz e Taylor, 2000)

$$
T(x, y)=x(1-x) y(1-y) \arctan \left[\alpha\left(\rho-\rho_{0}\right)\right],
$$

onde $\rho=(x+y) / \sqrt{2}, \quad \rho_{0}=0,8$ e $\alpha=20$. A Fig. 1 representa a solução exata em termos de temperatura.

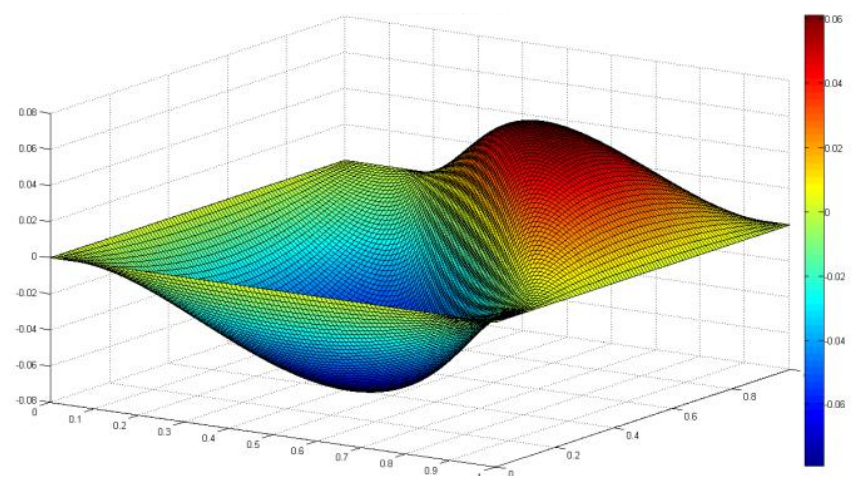

Figura 1. Problema 1 - solução analítica.

Pode-se perceber que a solução apresenta uma alta variação da temperatura em determinado trecho do domínio, o que, por sua vez, representa uma alta variação no gradiente, ou seja, no fluxo de calor dessa região. Para o corrente problema busca-se um erro relativo percentual admissível de $10 \%$ da energia total. Através da Fig. 2 observa-se a sequência de malhas geradas pelo processo adaptativo utilizando os vários estimadores propostos. Ademais, a Tab. 1 apresenta os parâmetros que caracterizam o processo adaptativo associado às malhas geradas.

Tabela 1. Resultados numéricos para o problema 1 - Erro relativo percentual global $(\eta)$, índice de efetividade $(\theta)$ e número de graus de liberdade (NGL).

\begin{tabular}{ccccc}
\hline Estimador & & Malha 1 & Malha 2 & Malha 3 \\
\hline Média Nodal & $\eta$ & $59,05 \%$ & $18,99 \%$ & $9,63 \%$ \\
Simples & $\theta$ & 0,6617 & 1,0382 & 1,0500 \\
& $N G L$ & 25 & 344 & 896 \\
\hline \multirow{3}{*}{ Zienkiewicz e Zhu } & $\eta$ & $55,81 \%$ & $16,91 \%$ & $10,20 \%$ \\
& $N G L$ & 0,6082 & 0,9626 & 0.9775 \\
& $\eta$ & 25 & 351 & 720 \\
\hline \multirow{2}{*}{ Superconvergent } & $\eta$ & $64,24 \%$ & $17,96 \%$ & $9,57 \%$ \\
Patch Recovery & $N G L$ & 0,7581 & 1,0828 & 1,0536 \\
& 25 & 429 & 991 \\
\hline
\end{tabular}

Observa-se que, para o problema em análise, todos os estimadores de erro apresentam um índice de efetividade tendendo a um quando o erro na malha tende a um valor baixo, característica essa que garante que o cálculo do erro efetuado pelo estimador, e que guia o processo adaptativo, é assintoticamente exato. Também é possível perceber que o erro relativo percentual global na última malha, para todos os estimadores, é muito próximo do erro relativo percentual admissível requerido em apenas duas iterações. Analisando o custo computacional, para o corrente problema, o Estimador $\mathrm{ZZ}$ mostra-se mais efetivo, pois alcança o erro requerido com um número de graus de liberdade inferior aos demais estimadores.

Pode-se, ainda, estabelecer uma comparação com os resultados apresentados por Castellazzi et al.(2010), onde o autor realiza adaptatividade isotrópica utilizando o software GID para a geração da malha e empregando um estimador de erro a posteriori baseado em recuperação por compatibilidade em padrões. Os resultados apresentados por Castellazzi et al.(2010) na malha 3 apresentam um índice de efetividade de 1,027, erro relativo percentual global de 9,20\% e 1881 elementos. Ou seja, estes resultados são muito próximos aos apresentados pelos estimadores aqui discutidos, exceto pelo número de elementos obtidos pelo Estimador ZZ. 


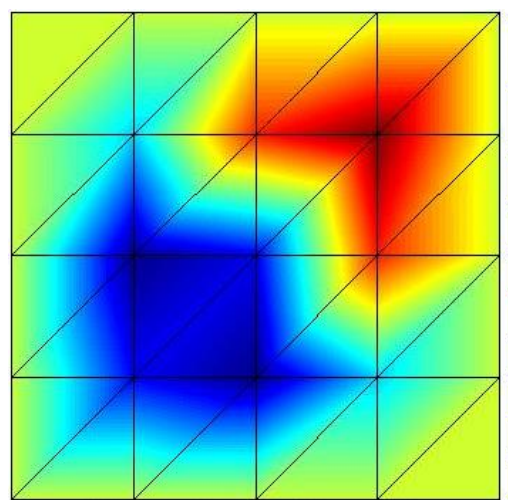

Malha a1 - 32 elementos

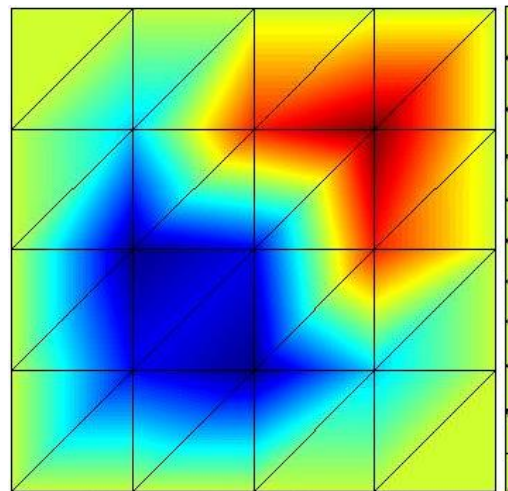

Malha b1 - 32 elementos

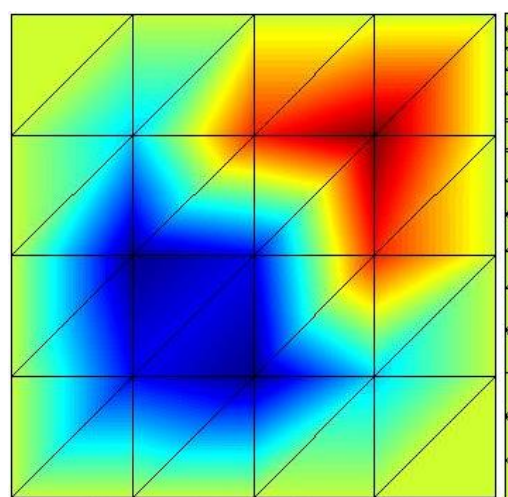

Malha c1 - 32 elementos

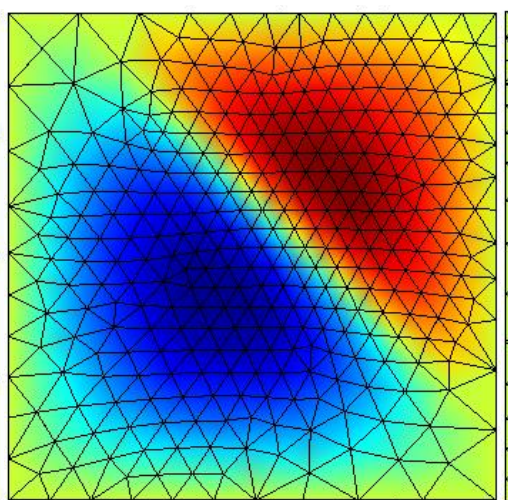

Malha a2 - 642 elementos

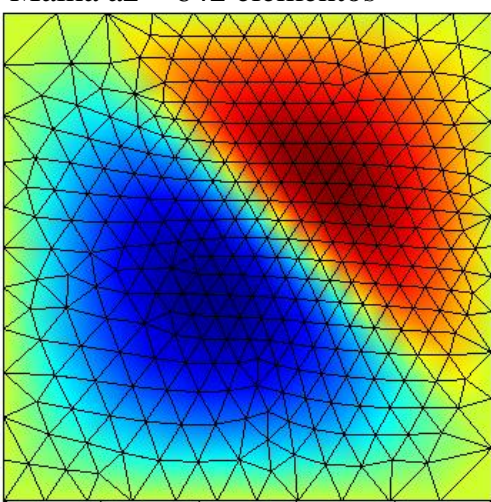

Malha b2 - 652 elementos

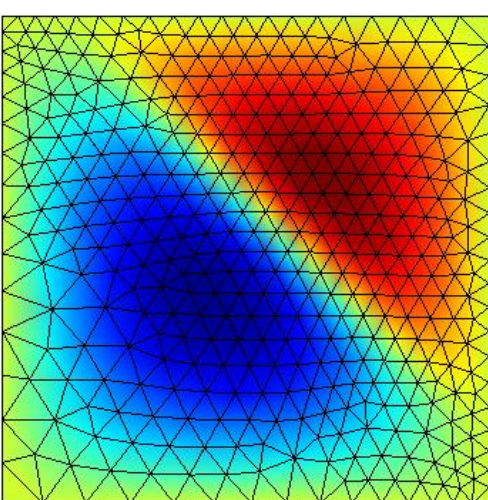

Malha c2 - 786 elementos

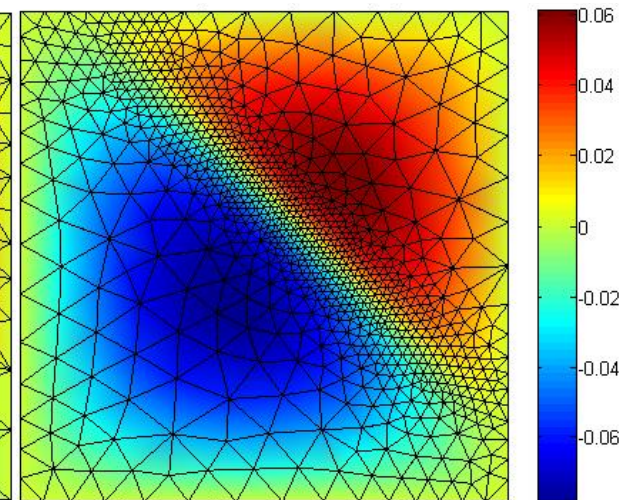

Malha a3 - 1724 elementos

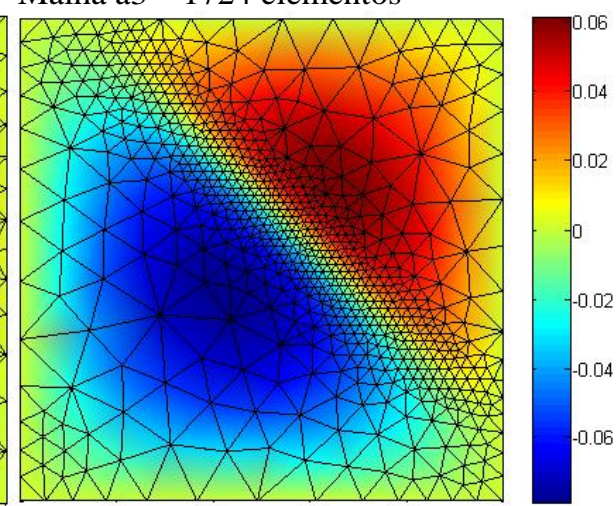

Malha b3 - 1380 elementos

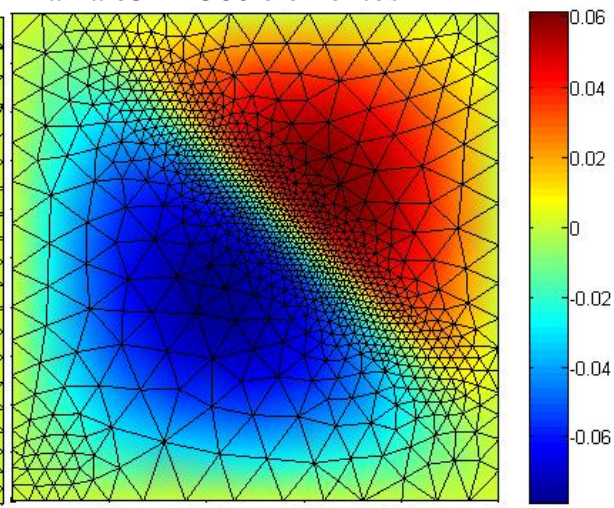

Malha c3 - 1917 elementos

Figura 2. Problema 1 - soluções adaptativas para (a) Método das Médias Nodais. (b) Estimador ZZ. (c) Estimador SPR.

\subsection{Problema 2}

Neste problema, o problema proposto por Mitchell (2013) é adaptado, sendo que a fonte interna de calor empregada é especificada de modo que a solução analítica seja

$$
T(x, y)=2^{2(a+b)} x^{a}(1-x)^{a} y^{b}(1-y)^{b},
$$

onde $a=20$ e $b=1$. Neste caso o erro relativo percentual admissível é de $5 \%$ da energia total. Após a aplicação dos diferentes estimadores em conjunto com o processo adaptativo, as malhas geradas são apresentadas na Fig. 3, confrontando-se os resultados numéricos por meio da Tab. 2. Percebe-se que, para a malha final, os números de graus de liberdade alcançados pelo processo adaptativo guiado pelos diferentes estimadores são muito próximos. Por outro lado é possível verificar uma determinada característica anisotrópica da malha devido à orientação da malha inicial, a qual se mantém principalmente na malha 2 utilizando o Estimador ZZ e o Método das MNS. Esta característica é menos acentuada na malha 3 dos diversos estimadores. Nesse sentido, o Estimador SPR mostrou-se superior apresentando uma melhor uniformidade da malha. A Fig. 4 representa a solução exata em termos de temperatura. 


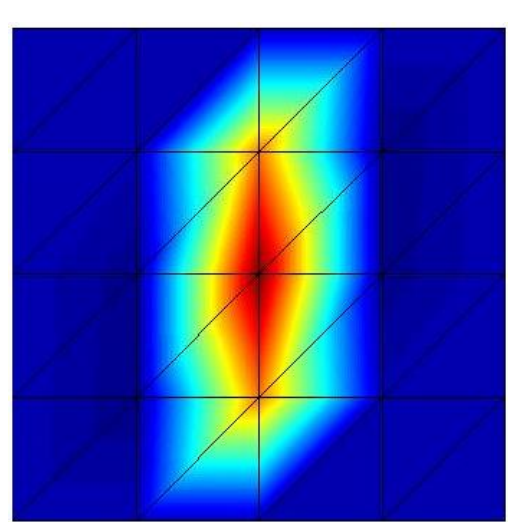

Malha a1 - 32 elementos

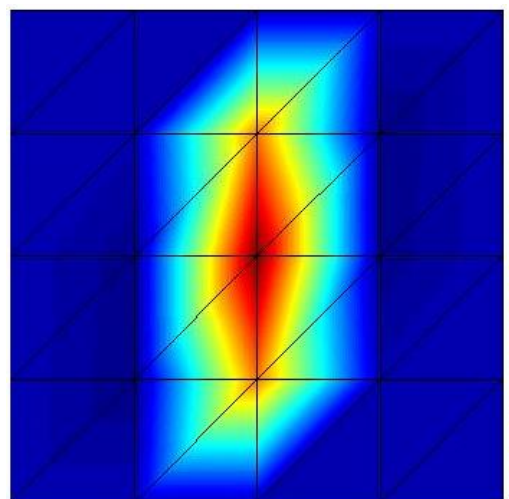

Malha b1 - 32 elementos

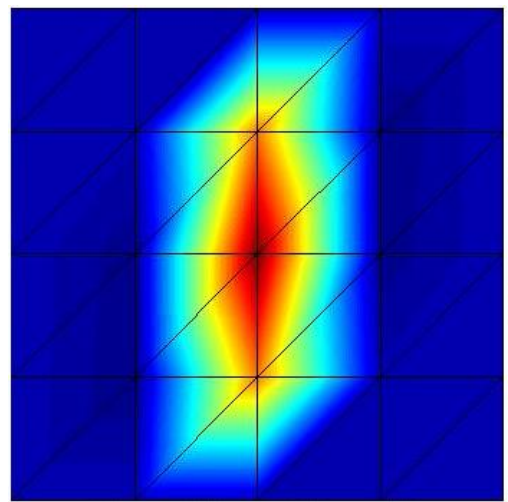

Malha c1 - 32 elementos

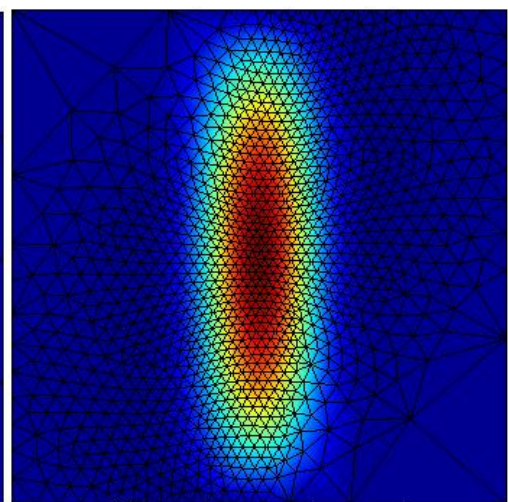

Malha a2 -2328 elementos

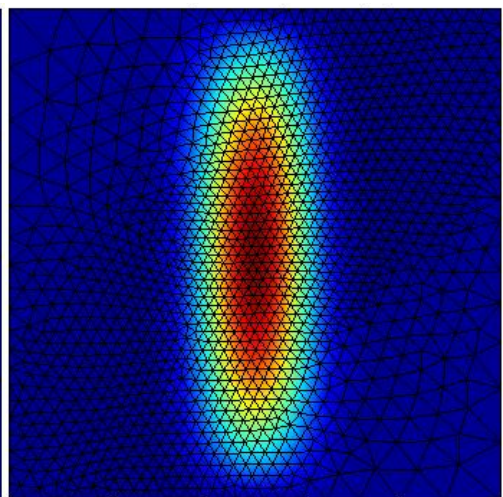

Malha b2 - 2750 elementos

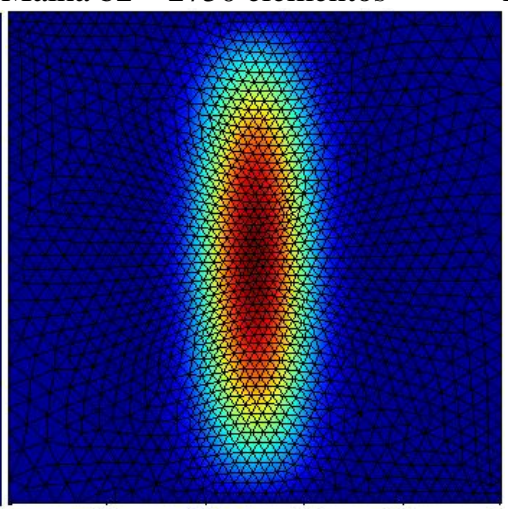

Malha c2 -2938 elementos

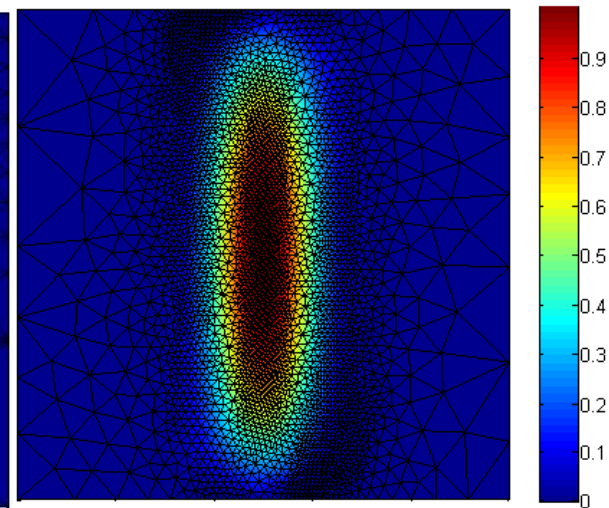

Malha a3 -7426 elementos

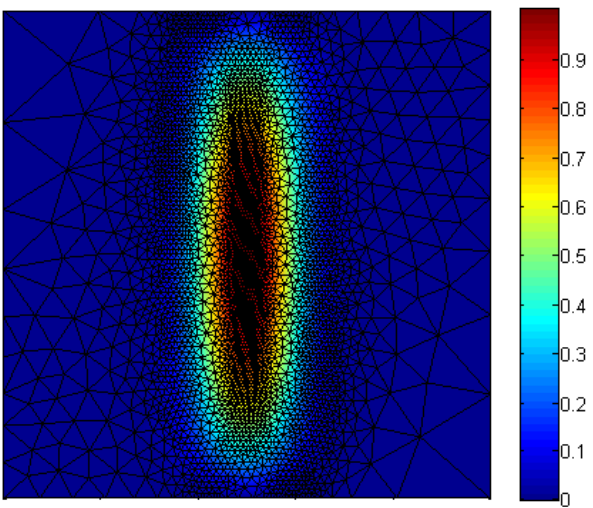

Malha b3 - 7715 elementos

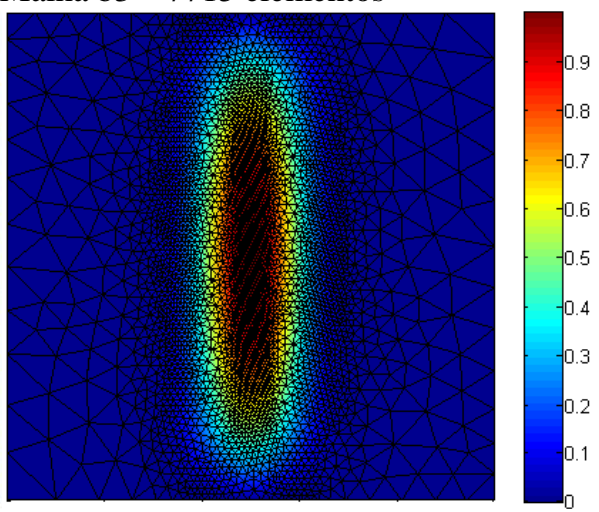

Malha c3-7782 elementos

Figura 3. Problema 2 - soluções adaptativas para (a) Método das Médias Nodais. (b) Estimador ZZ. (c) Estimador SPR.

Tabela 2. Resultados numéricos para o problema 2 - Erro relativo percentual global $(\eta)$, índice de efetividade $(\theta)$ e número de graus de liberdade (NGL).

\begin{tabular}{ccccc}
\hline Estimador & & Malha 1 & Malha 2 & Malha 3 \\
\hline \multirow{2}{*}{ Média Nodal } & $\eta$ & $62,34 \%$ & $10,65 \%$ & $4,68 \%$ \\
Simples & $\theta$ & 1,0438 & 1,0528 & 1,0415 \\
& $N G L$ & 25 & 1194 & 3758 \\
\hline \multirow{2}{*}{ Zienkiewicz e Zhu } & $\eta$ & $59,02 \%$ & $9,29 \%$ & $4,29 \%$ \\
& $\theta$ & 0,9572 & 0,9989 & 0,9973 \\
& $N G L$ & 25 & 1420 & 3898 \\
\hline \multirow{2}{*}{ Superconvergent } & $\eta$ & $64,36 \%$ & $8,96 \%$ & $4,37 \%$ \\
Patch Recovery & $\theta$ & 1,1008 & 1,0233 & 1,0429 \\
& $N G L$ & 25 & 1528 & 3934 \\
\hline
\end{tabular}




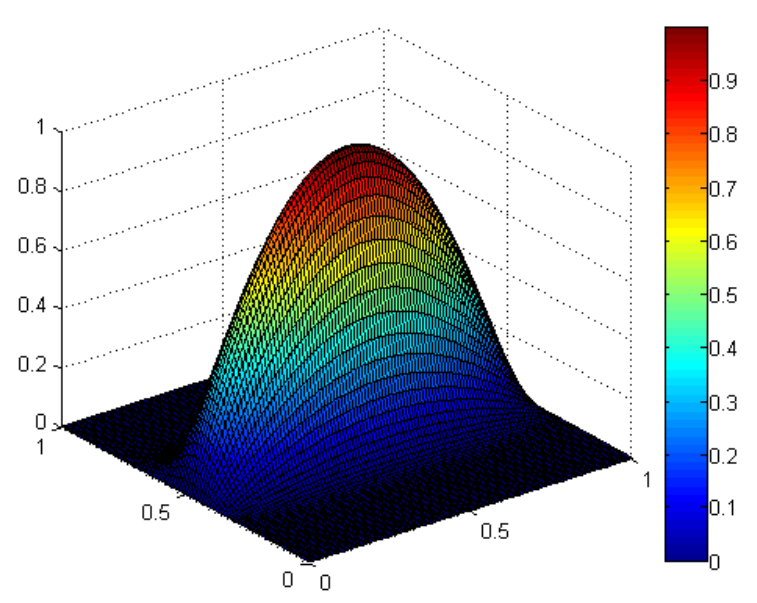

Figura 4. Problema 2 - solução analítica.

\section{CONCLUSÕES}

O corrente trabalho tem como objetivo a discussão sobre erros de aproximação no MEF, suas estimativas e adaptatividade da malha para o problema plano de condução térmica empregando o elemento linear CST. Foram utilizados três diferentes estimadores de erro a posteriori: MNS, ZZ e SPR, os quais serviram como guia para o processo h-adaptativo isotrópico descrito. A eficiência desses estimadores foi medida através do índice de efetividade, onde todos mostraram ótimos resultados. Ou seja, seus índices de efetividade tenderam a um quando o erro na malha tende a zero para números de graus de liberdade muito parecidos. Além disso, pode-se verificar que com a abordagem tomada foram necessárias no máximo duas iterações para que o erro relativo percentual admissível fosse alcançado, sendo que a primeira iteração em todos os casos alcança uma redução significativa do erro. Assim, conclui-se que o processo adaptativo descrito, quando conduzido por qualquer um dos estimadores apresentados, mostra-se efetivo e conduz a uma malha acompanhando os erros, ou seja, regiões com maior variação dos gradientes são mais refinadas. Outra característica importante desse processo é que o erro admissível é alcançado não somente pelo refino da malha, mas permite também seu desrefino. Esta propriedade geralmente leva a um custo computacional reduzido quando comparado com outras técnicas de geração de malha como mesh enrichment, na qual, sempre que o parâmetro de refinamento $\xi$ é maior que um, reduz o tamanho do elemento pela metade. Como futuros trabalhos, podem-se utilizar estimativas de erro e processos adaptativos anisotrópicos, os quais levam em consideração diferentes fatores dos abordados pelos estimadores aqui explanados.

\section{AGRADECIMENTOS}

Aos recursos e instrumentos proporcionados pelo Programa de Pós Graduação em Engenharia Mecânica - UFPR (PGMEC), CAPES, Programa Interdisciplinar em Engenharia do Petróleo e Gás Natural da UFPR (PRH24) e Instituto Federal do Paraná (IFPR).

\section{REFERÊNCIAS}

Ainsworth, M., Oden, J. T. 2000. A posteriori error estimation in finite element analysis. USA: Ed. John Wiley and Sons. 240 p.

Castellazzi, G., de Miranda, S. and Ubertini, F. 2010. Adaptivity based on the recovery by compatibility in patches. Finite Elements in Analysis and Design, 46, 379-390.

Ciarlet, P. G.2002. The Finite Element Method for Elliptic Problems. Paris: Ed. Elsevier. 530 p.

Cook, R. D., Malkus, D. S., Plesha, M. E., Witt, R. J. 2002. Concepts and Applications of Finite Element Analysis. Madison, USA: Ed. John Wiley and Sons. 719 p.

Fancello, E. A., Guimarães, A. C. e Feijóo, R. A. 1990. Aranha - Gerador de malhas 2D para elementos finitos triangulares de 3 e 6 nós. Anais do XI Congresso Ibero-Latino-Americano em Métodos Numéricos em Engenharia, vol. 2, (pp. 983-996). Brazil.

Fancello, E. A. e Feijóo, R. A. 1992. Adapte: estimador de erro para problemas planos em elasticidade linear. Mecânica Computacional, vol. 13. Bariloche, Argentina.

Mitchell, W. F. 2013. A collection of 2D elliptic problems for testing adaptive grid refinement algorithms. Applied Mathematics and Computation, 220, 350-364.

Prudhomme, S., Oden, J. T., Westermann, T., Bass, J. and Botkin, M. E. 2003. Practical methods for a posteriori error estimation in engineering applications. International Journal for Numerical Methods in Engineering, 56, 11931224.

Reddy, J. N. 2006. An Introduction to the Finite Element Method. Texas, USA: Ed. Mc Graw Hill. 766 p. 
Wiberg, N. E. and Abdulwahab, F. 1997. Error estimation with postprocessed finite element solutions. Computers \& Structures, 64, 113-137.

Zhu, J. Z. and Zienkiewicz, O. C. 1988. Adaptive techniques in the finite element method. Communications in Applied Numerical Methods, 4, 197-204.

Zienkiewicz, O. C., Taylor, R. L. 2000. The Finite Element Method. Oxford, USA: Ed. Butterworth Heinemann. 689 p.

Zienkiewicz, O. C., Taylor, R. L. 1989. The Finite Element Method - vol.1: Basic Formulation and Linear Problems. Maidenhead, England: Ed. Ed. Mc Graw Hill. 648 p.

Zienkiewicz, O. C. and Zhu, J. Z. 1987. A simple error estimator and adaptive procedure for practical engineering analysis. International Journal for Numerical Methods in Engineering, 24, 333-357.

Zienkiewicz, O. C. and Zhu, J. Z. 1992. The superconvergent patch recovery and a posteriori error estimates, Part 1: The recovery technique. International Journal for Numerical Methods in Engineering, 33, 1331-1364.

\title{
RESPONSABILIDADE AUTORAL
}

Os autores são os únicos responsáveis pelo conteúdo deste trabalho.

\section{ANALYSIS OF DIFFERENT A POSTERIORI ERROR ESTIMATORS APPLIED IN TWO-DIMENSIONAL PROBLEMS USING H-ADAPTIVE REFINEMENT}

\author{
Jéderson da Silva, dasilva.jederson@gmail.com ${ }^{1}$ \\ Francielly Elizabeth de Castro Silva, franciellye.castro@gmail.com ${ }^{1}$ \\ Jucélio Tomás Pereira, jucelio.tomas@ufpr.br ${ }^{1}$ \\ João do Carmo Lopes Gonçalves, joao.carmo@ifpr.edu.br ${ }^{1,2}$
${ }^{1}$ Federal University of Paraná - Postgraduate Program in Mechanical Engineering (PG-Mec) - Block IV, Department of Technology, Polytechnic Center UFPR, Jardim das America, Curitiba, Brazil.
2 Federal Institute of Paraná - Campus Paranaguá, Porto Seguro, Paranaguá, Brazil.

\begin{abstract}
The development of efficient methods for the generation of adaptive meshes for finite element analysis has been the focus of intensive research in recent decades. A reliable estimate of the approximation error of this numerical method is needed to guide properly an adaptive process. This work discusses the analysis of approximation errors, estimates and adaptivity of the mesh when applying the Finite Element Method (FEM) for two-dimensional problems using linear triangular element CST (Constant Strain Triangle). The a posteriori error estimation is based on the recovery of higher order derivatives and on the energy norm. The following estimators are used: estimator based on Nodal Simple Average(MNS), Zienkiewicz and Zhu (ZZ) estimator and Superconvergent Patch Recovery (SPR) estimator. In all the discussed problems it is employed as a measure of the quality of the estimators the concept of indexes of effectiveness. From calculating the error in each element, it is used an h-adaptive process to generate appropriate meshes to the problem in study, which aim an approximately uniform error, not exceeding the admissible error. It is discussed the theory inherent to the estimation error and the basic principles of the adopted h-adaptive process. Through numerical examples, it is performed a comparative analysis between different estimators and their efficiency in the context of an h-adaptive process.
\end{abstract}

Keywords: Finite Element Method, H-adaptivity, a posteriori error estimators, Superconvergent Patch Recovery Estimator, Zienkiewicz and Zhu (ZZ) Estimator. 